\title{
ON A CERTAIN CLASS OF CONCAVE MEROMORPHIC HARMONIC FUNCTIONS DEFINED BY INVERSE OF INTEGRAL OPERATOR
}

\author{
Yusuf AbDulahi And Maslina Darus
}

ABstRact. In this paper we introduce new class of meromorphic harmonic concave functions defined by an integral operator and establish some of the properties of this class.

2010 Mathematics Subject Classification: 30C45.

Keywords: Harmonic functions, Concave Univalent function, Jung-Kim-Srivastava integral operator, Salagean Operator.

\section{INTRODUCTION}

Let $A$ denotes the class of analytic functions of the form

$$
f(z)=z+\sum_{k=2}^{\infty} a_{k} z^{k}
$$

that map the unit disc conformally onto a domain whose complement with respect to a convex that satisfies the normalization $f(1)=\infty$, the opening angle of $f(U)$ at infinity is less than or equal to $\alpha \pi$.

The families of these functions is referred to as a concave univalent function denoted as $C_{o}(\alpha)$ if it satisfies the condition $P_{f}>0$, where

$$
P_{f}=\frac{2}{\alpha-1}\left[\frac{\alpha+1}{2} \frac{1+z}{1-z}-1-z \frac{f^{\prime \prime}(z)}{f^{\prime}(z)}\right]
$$

In [11], the concept of meromorphic concave function was introduced, that a conformal mapping of meromorphic functions on the unit disc is referred to as a 
concave function if its image is the complement of a compact convex function. We define the class of meromorphic functions to be of the form

$$
f(z)=\frac{1}{z}+\sum_{k=1}^{\infty} a_{k} z^{k}
$$

A function of the form (2) is referred to as a concave meromorphic if it satisfied the condition

$$
1+\operatorname{Re}\left[\frac{z f^{\prime \prime}(z)}{f^{\prime}(z)}\right]<0 .
$$

The concept of harmonic univalent function was first introduced by Clunie and Sheil-Small in [5]. The class of function is applied in the study of minimal surfaces and other areas of sciences. We say that a continuous function $f=u+i v$ is complex harmonic function in a domain $\mathbb{U} \subset \mathbb{C}$, if both $\mathrm{u}$ and $\mathrm{v}$ are real harmonic in $\mathbb{U}$. Let $f=h+\bar{g}$, where $h$ and $g$ are analytic in $\mathbb{U}$. A necessary and sufficient condition for $f$ to be locally univalent and preserving in $\mathbb{U}$ is that $\left|h^{\prime}(z)\right|>\left|g^{\prime}(z)\right|$ in $\mathbb{U}$.

In [6], it was shown that a complex valued, harmonic, sense preserving, univalent mapping $f$ must admit the representation

$$
f(z)=h(z)+\overline{g(z)}+A \log |z|
$$

where $h(z)$ and $g(z)$ are defined by

$$
h(z)=\alpha z+\sum_{k=1}^{\infty} a_{k} z^{-n}, g(z)=\beta \bar{z}+\sum_{k=1}^{\infty} b_{n} z^{-n}
$$

for $0 \leq|\beta|<|\alpha|$. In [7], For $z \in \mathbb{U}$. $S_{H}$ was define to be the class of functions

$$
f(z)=h(z)+\overline{g(z)}=\frac{1}{z}+\sum_{k=1}^{\infty} a_{k} z^{k}+\overline{\sum_{k=1}^{\infty} b_{k} z^{k}}
$$

which are harmonic in the unit disc $\mathbb{U}, h(z)$ and $g(z)$ are analytic in $\mathbb{U}$, respectively.

A function $f \in S_{H}$ is said to be in the subclass $S_{H}^{*}$ of meromorphic harmonic starlike functions in $\mathbb{U}$, if its satisfied the condition

$$
\operatorname{Re}\left[-\frac{z h^{\prime}(z)-\overline{z g^{\prime}(z)}}{h(z)+g(z)}\right]>0,(z \in \mathbb{U} \backslash 0) .
$$


Yusuf Abdulahi and Maslina Darus - On a Certain Class of Concave ...

Also A function $f \in S_{H}$ is said to be in the subclass $S C_{H}$ of meromorphic harmonic convex functions in $(U \backslash 0)$, if its satisfied the condition

$$
\operatorname{Re}\left[-\frac{z h^{\prime \prime}(z)+h^{\prime}(z) \overline{-z g^{\prime \prime}(z)+g^{\prime}(z)}}{h^{\prime}(z)-g^{\prime}(z)}\right]>0,(z \in \mathbb{U} \backslash 0) .
$$

These two classes ware studied in $[8,9,10]$.

In [12], the integral operator was introduced as the following:

$$
\mathcal{L}_{\sigma, \gamma} f(z)=\int_{0}^{z} \frac{(\gamma+1)^{2} t^{\sigma-1}}{z^{\gamma} \Gamma(\sigma)}\left(\log \frac{z}{t}\right)^{\sigma-1} f(t) d t
$$

and expressed as

$$
\mathcal{L}_{\sigma, \gamma} f(z)=z+\sum_{k=2}^{\infty}\left(\frac{\gamma+1}{\gamma+k}\right) a_{k} z^{k}
$$

In [13], the inverse of the integral operator was considered as

$$
\mathcal{J}_{\sigma, \gamma} f(z)=\int_{0}^{z} \frac{(\gamma+1)^{2} t^{\sigma-1}}{z^{\gamma} \Gamma(\sigma)}\left(\log \frac{z}{t}\right)^{-(\sigma-1)} f(t) d t
$$

an expressed as

$$
\mathcal{L}_{\sigma, \gamma} f(z)=z+\sum_{k=2}^{\infty}\left(\frac{\gamma+k}{\gamma+1}\right)^{\sigma} a_{k} z^{k}
$$

so that

$$
\mathcal{L}_{\sigma, \gamma}\left(\mathcal{J}_{\sigma, \gamma} f(z)\right)=f(z) .
$$

If $\gamma=0, n=\sigma$ we have $D^{n} f(z)$, known as the Salagean operator.

In this work, we studied a new class of meromorphic concave functions defined by inverse of an integral operator denoted $S H_{\gamma}^{\sigma} C_{0}$ and define the class as follows:

Definition 1 Let $S H_{\gamma}^{\sigma} C_{0}$ denote the class of meromorphic harmonic concave function define by inverse of Integral Operator on the function of the form (2)

$$
\mathcal{L}_{\sigma, \gamma} f(z)=\frac{1}{z}+\sum_{k=1}^{\infty}\left(\frac{\gamma+k}{\gamma+1}\right)^{\sigma} a_{k} z^{k}+\sum_{k=1}^{\infty}\left(\frac{\gamma+k}{\gamma+1}\right)^{\sigma} \overline{b_{k} z^{k}},(\sigma>0, \gamma>1)
$$

such that

$$
\operatorname{Re}\left[1+\frac{z\left(\mathcal{L}_{\sigma, \gamma} f(z)\right)^{\prime}}{\mathcal{L}_{\sigma, \gamma} f(z)}\right]<0
$$




\section{Main Results}

\subsection{Coefficient Inequalities for the class $S H_{\gamma}^{\sigma} C_{0}$}

Theorem 1. Let $\mathcal{L}_{\sigma, \gamma} f=h+g$ be of the form (11), if

$$
\sum_{k=1}^{\infty} k^{2}\left(\frac{\gamma+k}{\gamma+1}\right)^{\sigma}\left(\left|a_{n}\right|+\left|b_{n}\right|\right) \leq 1
$$

then $f$ is harmonic univalent, sense preserving in $U$

Proof. For $0<\left|z_{1}\right| \leq\left|z_{2}\right|<1$, we have

$$
\begin{aligned}
& \mid \mathcal{L}_{\sigma, \gamma} f\left(z_{1}\right)- \mathcal{L}_{\sigma, \gamma} f\left(z_{2}\right)|=| \frac{1}{z}+\sum_{k=1}^{\infty}\left(\frac{\gamma+k}{\gamma+1}\right)^{\sigma} a_{k} z_{1}^{k}+\sum_{k=1}^{\infty}\left(\frac{\gamma+k}{\gamma+1}\right)^{\sigma} \overline{b_{k} z_{1}^{k}} \\
&- \frac{1}{z}-\sum_{k=1}^{\infty}\left(\frac{\gamma+k}{\gamma+1}\right)^{\sigma} a_{k} z_{2}^{k}-\sum_{k=1}^{\infty}\left(\frac{\gamma+k}{\gamma+1}\right)^{\sigma} \overline{b_{k} z_{2}^{k} \mid} \\
& \geq \frac{1}{\left|z_{1}\right|}-\frac{1}{\left|z_{2}\right|}-\sum_{k=1}^{\infty}\left(\frac{\gamma+k}{\gamma+1}\right)^{\sigma}\left|a_{k}\right|\left|z_{1}^{k}-z_{2}^{k}\right|-\sum_{k=1}^{\infty}\left(\frac{\gamma+k}{\gamma+1}\right)^{\sigma}\left|b_{k}\right|\left|z_{1}^{k}-z_{2}^{k}\right| \\
&>\frac{\left|z_{1}-z_{2}\right|}{\left|z_{1} z_{2}\right|}-\left|z_{1}-z_{2}\right| \sum_{k=1}^{\infty} k\left(\frac{\gamma+k}{\gamma+1}\right)^{\sigma}\left(\left|a_{k}\right|+\left|b_{k}\right|\right) \\
&>\frac{\left|z_{1}-z_{2}\right|}{\left|z_{1} z_{2}\right|}\left[1-\left|z_{2}\right|^{2} \sum_{k=1}^{\infty} k^{2}\left(\frac{\gamma+k}{\gamma+1}\right)^{\sigma}\left(\left|a_{k}\right|+\left|b_{k}\right|\right)\right] \\
&>\frac{\left|z_{1}-z_{2}\right|}{\left|z_{1} z_{2}\right|}\left[1-\sum_{k=1}^{\infty} k^{2}\left(\frac{\gamma+k}{\gamma+1}\right)^{\sigma}\left(\left|a_{k}\right|+\left|b_{k}\right|\right)\right]
\end{aligned}
$$

The last expression is non negative by $\sum_{k=1}^{\infty} k^{2}\left(\frac{\gamma+k}{\gamma+1}\right)^{\sigma}\left(\left|a_{k}\right|+\left|b_{k}\right|\right)<1, \mathcal{L}_{\sigma, \gamma} f(z)$ is univalent in $\mathrm{U}$.

Now we want to show that $f$ is sense preserving in $U$, we need to show that $\left|h^{\prime}(z)\right| \geq\left|g^{\prime}(z)\right|$ in $U$,

$$
\begin{gathered}
\left|h^{\prime}(z)\right| \geq \frac{1}{|z|^{2}}-\sum_{k=1}^{\infty} k\left(\frac{\gamma+k}{\gamma+1}\right)^{\sigma}\left|a_{k}\right||z|^{k-1} \\
=\frac{1}{r^{2}}-\sum_{k=1}^{\infty} k\left(\frac{\gamma+k}{\gamma+1}\right)^{\sigma}\left|a_{k}\right| r^{k-1}
\end{gathered}
$$




$$
\begin{gathered}
>1-\sum_{k=1}^{\infty} k\left(\frac{\gamma+k}{\gamma+1}\right)^{\sigma}\left|a_{k}\right| \\
\geq 1-\sum_{k=1}^{\infty} k^{2}\left(\frac{\gamma+k}{\gamma+1}\right)^{\sigma}\left|a_{k}\right| \\
\geq \sum_{k=1}^{\infty} k^{2}\left(\frac{\gamma+k}{\gamma+1}\right)^{\sigma}\left|b_{k}\right| \\
>\sum_{k=1}^{\infty} k\left(\frac{\gamma+k}{\gamma+1}\right)^{\sigma}\left|b_{k}\right| r^{k-1}=\sum_{k=1}^{\infty} k\left(\frac{\gamma+k}{\gamma+1}\right)^{\sigma}\left|b_{k}\right||z|^{k-1} \geq\left|g^{\prime}(z)\right| .
\end{gathered}
$$

Thus this completes the proof of the theorem.

Theorem 2. Let $\mathcal{L}_{\sigma, \gamma} f=h+g$ be of the form (11), then $f \in S H_{\gamma}^{\sigma} C_{0}$ if the condition holds

$$
\sum_{k=1}^{\infty} k^{2}\left(\frac{\gamma+k}{\gamma+1}\right)^{\sigma}\left(\left|a_{n}\right|+\left|b_{n}\right|\right) \leq 1
$$

Proof. with the condition that Rew $<0 \leftrightarrow\left|\frac{w+1}{w-1}\right|<1$, it suffices to show that $\left|\frac{w+1}{w-1}\right|<1$.

Let

$$
w=R e\left[1+\frac{z\left(\mathcal{L}_{\sigma, \gamma} f(z)\right)^{\prime}}{\mathcal{L}_{\sigma, \gamma} f(z)}\right]
$$

such that $w=\frac{z g^{\prime}(z)}{g(z)}$, where $g(z)=z\left(\mathcal{L}_{\sigma, \gamma} f(z)\right)^{\prime}$ we have that

$$
\begin{gathered}
\left|\frac{w+1}{w-1}\right|=\left|\frac{\sum_{k=1}^{\infty}\left(k^{2}+k\right)(\gamma+k / \gamma+1) a_{k} z^{k}-\sum_{k=1}^{\infty}\left(k^{2}+k\right)(\gamma+k / \gamma+1) b_{k} z^{k}}{\frac{2}{z}+\sum_{k=1}^{\infty}\left(k^{2}+k\right)(\gamma+k / \gamma+1) a_{k} z^{k}-\sum_{k=1}^{\infty}\left(k^{2}+k\right)(\gamma+k / \gamma+1) b_{k} z^{k}}\right| \\
<\frac{\sum_{k=1}^{\infty}\left(k^{2}+k\right)(\gamma+k / \gamma+1)\left|a_{k}\right|-\sum_{k=1}^{\infty}\left(k^{2}+k\right)(\gamma+k / \gamma+1)\left|b_{k}\right|}{2-\sum_{k=1}^{\infty}\left(k^{2}-k\right)(\gamma+k / \gamma+1)\left|a_{k}\right|-\sum_{k=1}^{\infty}\left(k^{2}-k\right)(\gamma+k / \gamma+1)\left|b_{k}\right|}
\end{gathered}
$$

The last expression is bounded above by 1 if

$$
\begin{gathered}
\sum_{k=1}^{\infty}\left(k^{2}+k\right)(\gamma+k / \gamma+1) a_{k}+\sum_{k=1}^{\infty}\left(k^{2}+k\right)(\gamma+k / \gamma+1) b_{k} \\
\leq 2-\sum_{k=1}^{\infty}\left(k^{2}-k\right)(\gamma+k / \gamma+1) a_{k}-\sum_{k=1}^{\infty}\left(k^{2}-k\right)(\gamma+k / \gamma+1) b_{k}
\end{gathered}
$$


which is equivalent to our condition by

$$
\sum_{k=1}^{\infty} k^{2}\left(\frac{\gamma+k}{\gamma+1}\right)^{\sigma}\left(\left|a_{n}\right|+\left|b_{n}\right|\right) \leq 1
$$

Conversely, assume $f \in S H_{\gamma}^{\sigma} C_{0}$, then we have

$$
\begin{gathered}
\left|\frac{1+\frac{\left.\left.z \mathcal{L}_{\sigma, \gamma} h(z)\right)^{\prime \prime}-z \mathcal{L}_{\sigma, \gamma} g(z)\right)^{\prime \prime}}{\left.\left.\mathcal{L}_{\sigma, \gamma} h(z)\right)^{\prime}-\mathcal{L}_{\sigma, \gamma} g(z)\right)^{\prime}}+1}{1+\frac{\left.\left.z \mathcal{L}_{\sigma, \gamma} h(z)\right)^{\prime \prime}-z \mathcal{L}_{\sigma, \gamma} g(z)\right)^{\prime \prime}}{\left.\left.\mathcal{L}_{\sigma, \gamma} h(z)\right)^{\prime}-\mathcal{L}_{\sigma, \gamma} g(z)\right)^{\prime}}-1}\right|<1 \\
=\left|\frac{\sum_{k=1}^{\infty}\left(k^{2}+k\right)(\gamma+k / \gamma+1) a_{k}-\sum_{k=1}^{\infty}\left(k^{2}+k\right)(\gamma+k / \gamma+1) \overline{b_{k}}}{\frac{2}{z^{2}}-\sum_{k=1}^{\infty}\left(k^{2}-k\right)(\gamma+k / \gamma+1) a_{k}-\sum_{k=1}^{\infty}\left(k^{2}-k\right)(\gamma+k / \gamma+1) \overline{b_{k}}}\right|<1 .
\end{gathered}
$$

By letting $|z| \rightarrow 1$, we obtain (12).

Theorem 3. Let $f=h+\bar{g}$ of the form (11), then a necessary and sufficient condition for $\mathcal{L}_{\sigma, \gamma} f(z)$ to be in $S H_{\gamma}^{\sigma} C_{0}$ is that

$$
\sum_{k=1}^{\infty} k^{2}\left(\frac{\gamma+k}{\gamma+1}\right)^{\sigma}\left(\left|a_{k}\right|+\left|b_{k}\right|\right) \leq 1
$$

Proof. From Theorem 2, we assume that

$$
\sum_{k=1}^{\infty} k^{2}\left(\frac{\gamma+k}{\gamma+1}\right)^{\sigma}\left(\left|a_{k}\right|+\left|b_{k}\right|\right)>1
$$

Since $\mathcal{L}_{\sigma, \gamma} f(z) \in \mathcal{L}_{\sigma, \gamma} S_{H} C_{0}$, then $1+\operatorname{Rez}\left(\mathcal{L}_{\sigma, \gamma} f(z)\right)^{\prime \prime} /\left(\mathcal{L}_{\sigma, \gamma} f(z)\right)^{\prime}$ is equivalent to

$$
\begin{gathered}
\operatorname{Re} \frac{z g^{\prime}(z)}{g(z)}=\operatorname{Re} \frac{z\left(\frac{1}{z^{2}}+\sum_{k=1}^{\infty} k^{2}\left(\frac{\gamma+k}{\gamma+1}\right)^{\sigma} a_{k} z^{k-1}+\sum_{k=1}^{\infty} k^{2}\left(\frac{\gamma+k}{\gamma+1}\right)^{\sigma} \overline{b_{k} z^{k-1}}\right)}{\frac{1}{z}+\sum_{k=1}^{\infty} k\left(\frac{\gamma+k}{\gamma+1}\right)^{\sigma} a_{k} z^{k}+\sum_{k=1}^{\infty} k\left(\frac{\gamma+k}{\gamma+1}\right)^{\sigma} \overline{b_{k} z^{k}}} \\
=\operatorname{Re} \frac{\left(\frac{1}{z}+\sum_{k=1}^{\infty} k^{2}\left(\frac{\gamma+k}{\gamma+1}\right)^{\sigma} a_{k} z^{k}+\sum_{k=1}^{\infty} k^{2}\left(\frac{\gamma+k}{\gamma+1}\right)^{\sigma} \overline{b_{k} z^{k}}\right)}{\frac{1}{z}+\sum_{k=1}^{\infty} k\left(\frac{\gamma+k}{\gamma+1}\right)^{\sigma} a_{k} z^{k}+\sum_{k=1}^{\infty} k\left(\frac{\gamma+k}{\gamma+1}\right)^{\sigma} \overline{b_{k} z^{k}}} \leq 0
\end{gathered}
$$

for $|z|=r>1$, the above expression reduce to

$$
\operatorname{Re}\left(\frac{1+\sum_{k=1}^{\infty} k^{2}\left(\frac{\gamma+k}{\gamma+1}\right)^{\sigma}\left(\left|a_{k}\right|+\left|b_{k}\right|\right) r^{k}}{1+\sum_{k=1}^{\infty} k\left(\frac{\gamma+k}{\gamma+1}\right)^{\sigma}\left(\left|a_{k}\right|+\left|b_{k}\right|\right) r^{k}}\right)=\left(\frac{A(r)}{B(r)}\right) \leq 0
$$


from our assumption that $\sum_{k=1}^{\infty} k^{2}\left(\frac{\gamma+k}{\gamma+1}\right)^{\sigma}\left(\left|a_{k}\right|+\left|b_{k}\right|\right)>1$, then $A(r)$ and $B(r)$ are positive for $r$ sufficiently close to 1 . Thus there exists a $z_{0}=r_{0}>1$ for which the quotient is positive. This contradicts the required condition that $\frac{A(r)}{B(r)} \leq 0$, so the proof is complete.

\subsection{Distortion and Extreme point}

Theorem 4. If $\mathcal{L}_{\sigma, \gamma} f_{k}=h_{k}+\overline{g_{k}}$ be of the form (11) and $0<|z|=r<1$, then

$$
\left|\mathcal{L}_{\sigma, \gamma} f_{k}(z)\right| \leq \frac{1+r^{2}}{r}
$$

and

$$
\left|\mathcal{L}_{\sigma, \gamma} f_{k}(z)\right| \leq \frac{1-r^{2}}{r}
$$

Proof. Taking the absolute of $f_{k}$, we have that

$$
\begin{aligned}
\left|f_{k}\right|=\mid \frac{1}{z} & +\sum_{k=1}^{\infty}\left(\frac{\gamma+k}{\gamma+1}\right)^{\sigma} a_{k} z^{k}+\sum_{k=1}^{\infty}\left(\frac{\gamma+k}{\gamma+1}\right)^{\sigma} \overline{b_{k} z^{k}} \mid \\
& \geq \frac{1}{r}-\sum_{k=1}^{\infty}\left(\frac{\gamma+k}{\gamma+1}\right)^{\sigma}\left(\left|a_{k}\right|+\left|b_{k}\right|\right) r^{k} \\
& \geq \frac{1}{r}-\sum_{k=1}^{\infty} k^{2}\left(\frac{\gamma+k}{\gamma+1}\right)^{\sigma}\left(\left|a_{k}\right|+\left|b_{k}\right|\right) r
\end{aligned}
$$

by applying $\sum_{k=1}^{\infty} k^{2}\left(\frac{\gamma+k}{\gamma+1}\right)^{\sigma}\left(\left|a_{k}\right|+\left|b_{k}\right|\right) \leq 1$

$$
\left|f_{k}\right| \geq \frac{1}{r}-r=\frac{1-r^{2}}{r} .
$$

Also

$$
\begin{aligned}
\left|f_{k}\right|=\mid \frac{1}{z} & +\sum_{k=1}^{\infty}\left(\frac{\gamma+k}{\gamma+1}\right)^{\sigma} a_{k} z^{k}+\sum_{k=1}^{\infty}\left(\frac{\gamma+k}{\gamma+1}\right)^{\sigma} \overline{b_{k} z^{k}} \mid \\
& \leq \frac{1}{r}+\sum_{k=1}^{\infty}\left(\frac{\gamma+k}{\gamma+1}\right)^{\sigma}\left(\left|a_{k}\right|+\left|b_{k}\right|\right) r^{k} \\
& \leq \frac{1}{r}+\sum_{k=1}^{\infty} k^{2}\left(\frac{\gamma+k}{\gamma+1}\right)^{\sigma}\left(\left|a_{k}\right|+\left|b_{k}\right|\right) r
\end{aligned}
$$


by applying $\sum_{k=1}^{\infty} k^{2}\left(\frac{\gamma+k}{\gamma+1}\right)^{\sigma}\left(\left|a_{k}\right|+\left|b_{k}\right|\right) \leq 1$

$$
\left|f_{k}\right| \leq \frac{1}{r}+r=\frac{1+r^{2}}{r}
$$

Theorem 5. Let $\mathcal{L}_{\sigma, \gamma} f=h+\bar{g}$ be of the form (11). Set $h_{n, 0}=g_{n, 0}=\frac{1}{z}$ for $k=1,2,3, \cdots$ set

$$
h_{n, k}(z)=\frac{1}{z}+\frac{1}{k^{2}} z^{k}, g_{n, k}(z)=\frac{1}{z}+\frac{1}{k^{2}} \overline{z^{k}}
$$

then $\mathcal{L}_{\sigma, \gamma} f(z)$ to be in $S H_{\gamma}^{\sigma} C_{0}$ if and only if $f_{k}$ can be expressed as

$$
f_{n, k}=\sum_{k=0}^{\infty}\left(\Psi_{k} h_{n, k}(z)+\Phi_{k} g_{n, k}(z)\right)
$$

where $\Psi_{k} \geq 0, \Phi_{k} \geq 0$ and $\sum_{k=0}^{\infty}\left(\Psi_{k}+\Phi_{k}\right)=1$.

Proof. For function $f=h+g$ to be of the form (11), we have that

$$
\begin{gathered}
f_{n, k}(z)=\sum_{k=1}^{\infty}\left(\Psi_{k} h_{n, k}(z)+\Phi_{k} g_{n, k}(z)\right) \\
=\Psi_{0} h_{n, 0}+\Phi_{0} g_{n, 0}+\sum_{k=1}^{\infty}\left(\Psi_{k} h_{n, k}(z)+\Phi_{k} g_{n, k}(z)\right) \\
=\Psi_{0} h_{n, 0}+\Phi_{0} g_{n, 0}+\sum_{k=1}^{\infty} \Psi_{k}\left(\frac{1}{z}+\frac{1}{k^{2}} z^{k}\right)+\sum_{k=1}^{\infty} \Phi_{k}\left(\frac{1}{z}+\frac{1}{k^{2}} \overline{z^{k}}\right) \\
\sum_{k=0}^{\infty}\left(\Psi_{k}+\Phi_{k}\right) \frac{1}{z}+\sum_{k=1}^{\infty} \frac{1}{k^{2}}\left(\Psi_{k} z^{k}+\Phi_{k} \overline{z^{k}}\right) .
\end{gathered}
$$

Now by Theorem 1 ,

$$
\sum_{k=1}^{\infty}\left(\Psi_{k} \frac{1}{k^{2}} k^{2}+\Phi_{k} \frac{1}{k^{2}} k^{2}\right)=\sum_{k=1}^{\infty} \Psi_{k}+\Phi_{k}=1-\Psi_{0}-\Phi_{0} \leq 1
$$

we have $\mathcal{L}_{\sigma, \gamma} f(z)$ to be in $S H_{\gamma}^{\sigma} C_{0}$. The converse is similar to the above proof 


\subsection{Convolution Properties}

For harmonic functions

$$
\mathcal{L}_{\sigma, \gamma} f_{n}(z)=\frac{1}{z}+\sum_{k=1}^{\infty}\left(\frac{\gamma+k}{\gamma+1}\right)^{\sigma} a_{k} z^{k}+\sum_{k=1}^{\infty}\left(\frac{\gamma+k}{\gamma+1}\right)^{\sigma} \overline{b_{k} z^{k}}
$$

and

$$
\mathcal{L}_{\sigma, \gamma} F_{n}(z)=\frac{1}{z}+\sum_{k=1}^{\infty}\left(\frac{\gamma+k}{\gamma+1}\right)^{\sigma} A_{k} z^{k}+\sum_{k=1}^{\infty}\left(\frac{\gamma+k}{\gamma+1}\right)^{\sigma} \overline{B_{k} z^{k}}
$$

The convolution of $\mathcal{L}_{\sigma, \gamma} f_{n}(z)$ and $\mathcal{L}_{\sigma, \gamma} F_{n}(z)$ is given by $\left(\mathcal{L}_{\sigma, \gamma} f_{n} * \mathcal{L}_{\sigma, \gamma} F_{n}\right)(z)=$ $\mathcal{L}_{\sigma, \gamma} f_{n}(z) * \mathcal{L}_{\sigma, \gamma} F_{n}(z)$

$$
=\frac{1}{z}+\sum_{k=1}^{\infty}\left(\frac{\gamma+k}{\gamma+1}\right)^{\sigma}\left|a_{k}\right|\left|A_{k}\right| z^{k}+\sum_{k=1}^{\infty}\left(\frac{\gamma+k}{\gamma+1}\right)^{\sigma}\left|b_{k}\right|\left|B_{k}\right| \overline{z^{k}} .
$$

The geometric convolution of $f_{k}$ and $F_{k}$ is given by

$$
\left(f(z) * F_{k}\right)(z)=f_{k}(z) \bullet F_{k}(z)=\frac{1}{z}+\sum_{k=1}^{\infty}\left(\frac{\gamma+k}{\gamma+1}\right)^{\sigma} \sqrt{\left|a_{k} A_{k}\right|} z^{k}+\sum_{k=1}^{\infty}\left(\frac{\gamma+k}{\gamma+1}\right)^{\sigma} \sqrt{\left|b_{k} B_{k}\right| z^{k}} .
$$

The integral convolution of $f_{k}$ and $F_{k}$ is given by

$$
\left(f_{k} \circ F_{k}\right)(z)=f_{k}(z) \circ F_{k}(z)=\frac{1}{z}+\sum_{k=1}^{\infty}\left(\frac{\gamma+k}{\gamma+1}\right)^{\sigma} \frac{\left|a_{k} A_{k}\right|}{k} z^{k}+\sum_{k=1}^{\infty}\left(\frac{\gamma+k}{\gamma+1}\right)^{\sigma} \frac{\left|b_{k} B_{k}\right|}{k} z^{k}
$$

Theorem 6. Let $\mathcal{L}_{\sigma, \gamma} f_{k}(z) \in S H_{\gamma}^{\sigma} C_{0}$ and $\mathcal{L}_{\sigma, \gamma} F_{k}(z) \in S H_{\gamma}^{\sigma} C_{0}$. Then the convolution $\mathcal{L}_{\sigma, \gamma} f_{k}(z) * \mathcal{L}_{\sigma, \gamma} F_{k}(z) \in S H_{\gamma}^{\sigma} C_{0}$.

Proof. From (17), (18), then the convolution given by (19). We need to show that the coefficients of $\mathcal{L}_{\sigma, \gamma} f_{k}(z) * \mathcal{L}_{\sigma, \gamma} F_{k}(z)$ satisfy the condition of theorem (2.1). We obtain that

$$
\begin{gathered}
\sum_{k=1}^{\infty} k^{2}\left(\frac{\gamma+k}{\gamma+1}\right)^{\sigma}\left(\left|a_{k}\right|\left|A_{k}\right|\right)+\sum_{k=1}^{\infty} k^{2}\left(\frac{\gamma+k}{\gamma+1}\right)^{\sigma}\left(\left|b_{k}\right|\left|B_{k}\right|\right) \\
\leq \sum_{k=1}^{\infty} k^{2}\left(\frac{\gamma+k}{\gamma+1}\right)^{\sigma}\left|a_{k}\right|+\sum_{k=1}^{\infty} k^{2}\left(\frac{\gamma+k}{\gamma+1}\right)^{\sigma}\left|b_{k}\right| \leq 1 .
\end{gathered}
$$

Therefore $\mathcal{L}_{\sigma, \gamma} f_{k}(z) * \mathcal{L}_{\sigma, \gamma} F_{k}(z) \in \mathcal{L}_{\sigma, \gamma} S_{H} C_{0}$, where $\left|A_{k}\right| \leq 1,\left|B_{k}\right| \leq 1$. This completes the proof. 
Theorem 7. Given $f_{k}$ and $F_{k}$ of the form (17) and (18) belong to the class $S H_{\gamma}^{\sigma} C_{0}$, then the geometric condition $\left(f(z) \bullet F_{k}\right)(z) \in S H_{\gamma}^{\sigma} C_{0}$.

Proof. From (19), and by Cauchy-Schwartz's inequality, it follows that

$$
\sum_{k=1}^{\infty} k^{2}\left(\frac{\gamma+k}{\gamma+1}\right)^{\sigma}\left(\sqrt{\left|A_{k} a_{k}\right|}+\sqrt{\left|B_{k} b_{k}\right|}\right) \leq 1
$$

Theorem 8. Given $f_{k}$ and $F_{k}$ of form (17) and (18) belong to the class $S H_{\gamma}^{\sigma} C_{0}$, then the integral convolution $\left(f(z) \circ F_{k}\right)(z) \in S H_{\gamma}^{\sigma} C_{0}$.

Proof. Let $\left|A_{k}\right| \leq 1$ and $\left|B_{k}\right| \leq 1$, then

$$
\begin{gathered}
\sum_{k=1}^{\infty} k^{2}\left(\frac{\gamma+k}{\gamma+1}\right)^{\sigma}\left(\frac{\left|A_{k} a_{k}\right|}{k}+\frac{\left|B_{k} b_{k}\right|}{k}\right) \\
\leq \sum_{k=1}^{\infty} k^{2}\left(\frac{\gamma+k}{\gamma+1}\right)^{\sigma}\left(\frac{\left|a_{k}\right|}{k}+\frac{\left|b_{k}\right|}{k}\right) \\
\leq \sum_{k=1}^{\infty} k^{2}\left(\frac{\gamma+k}{\gamma+1}\right)^{\sigma}\left(\frac{\left|a_{k}\right|}{k}+\frac{\left|b_{k}\right|}{k}\right) \leq 1 .
\end{gathered}
$$

The proof is complete.

\subsection{Convex Combinations}

Theorem 9. The class $S H_{\gamma}^{\sigma} C_{0}$ is closed under convex combination.

Proof. Let $i=1,2, \cdots$, then

$$
\mathcal{L}_{\sigma, \gamma} f_{i}(z)=\frac{1}{z}+\sum_{k=1}^{\infty}\left(\frac{\gamma+k}{\gamma+1}\right)^{\sigma} a_{i k} z^{k}+\sum_{k=1}^{\infty}\left(\frac{\gamma+k}{\gamma+1}\right)^{\sigma} \overline{i b_{k} z^{k}}
$$

where $a_{i k}>0, b_{i k}>0$, by theorem $(2)$

$$
\sum_{k=1}^{\infty} k^{2}\left(\frac{\gamma+k}{\gamma+}\right)^{\sigma}\left(\left|a_{i k}\right|+\left|b_{i k}\right|\right) \leq 1 .
$$

For $\sum_{k=1}^{\infty} t_{i}=1,0 \leq t \leq 1$, the convex combinations of $\mathcal{L}_{\sigma, \gamma} f(z)$ is written as

$$
\sum_{k=1}^{\infty} t_{i} \mathcal{L}_{\sigma, \gamma} f_{i}(z)=\frac{1}{z}+\sum_{k=1}^{\infty}\left(\frac{\gamma+k}{\gamma+1}\right)^{\sigma}\left(t_{i} a_{i k}\right) z^{k}+\sum_{k=1}^{\infty}\left(\frac{\gamma+k}{\gamma+1}\right)^{\sigma} \overline{\left.t_{i} b_{i k}\right) z^{k}}
$$


Yusuf Abdulahi and Maslina Darus - On a Certain Class of Concave ...

Then by

$$
\begin{gathered}
\sum_{k=1}^{\infty} k^{2}\left(\frac{\gamma+k}{\gamma+1}\right)^{\sigma}\left(\left|a_{i k}\right|+\left|b_{i k}\right|\right) \leq 1 \\
\sum_{k=1}^{\infty} k^{2}\left(\frac{\gamma+k}{\gamma+1}\right)^{\sigma}\left[\mid \sum_{k=1}^{\infty}\left(t_{i} a_{i k}|+| \sum_{k=1}^{\infty} t_{i} b_{i k} \mid\right)\right] \\
=\sum_{k=1}^{\infty} t_{i}\left[\sum_{k=1}^{\infty} k^{2}\left(\frac{\gamma+k}{\gamma+1}\right)^{\sigma}\left(\left|a_{i k}\right|+\left|b_{i k}\right|\right)\right] \leq \sum_{k=1}^{\infty} t_{i}=1
\end{gathered}
$$

. Then $\sum_{k=1}^{\infty} t_{i} \mathcal{L}_{\sigma, \gamma} f_{i}(z) \in S H_{\gamma}^{\sigma} C_{0}$.

Acknowledgement: The second author is partially supported by UKM grant: GUP-2017-064.

\section{REFERENCES}

[1] I. AlDawish and M. Darus, On p-valent Concave Functions, Kochi Journal of Mathematics, 10(2015), 43-51.

[2] I. G. Avkhadiev and K. J Wirth, Concave schilct function with bounded opening angle at Infinity, Lobachevskii J. Math, 17(2005), 3-10.

[3] F.G. Avkhadiev and K.J. Wirths Concave schlict functions with bounded opening angle at infinity, Lobachevskii Journal of Mathematics 17:3-10.

[4] M. Chuaqui, P. Duren and B. Osgood, . Concave conformal mappings and prevertices of Schwarz-Christoffel mappings, Proceedings of the American Mathematical Society 140(2012) 3495-3505.

[5] J. Clunie and T. Sheil-Small Harmonic univalent functions. Annales Academiae Scientiarum Fennicae. Series A.I.Mathematica 9(1984.) 3-25.

[6] W. Hengartner and G. Schober . Univalent harmonic functions. Transactions of the American Mathematical Society 299(1987) 1-31.

[7] K. AL-Shaqsi and M. Darus On harmonic uinvalent functions with respect to k-symmetric points. International Journal of Contemporary Mathematical Sciences 3 (2008),111-118.

[8] J.M. Jahangiri and H. Silverman Meromorphic univalent harmonic functions with negative coefficients. Bulletin-Korean Mathematical Society 36(4), 1999,763-770.

[9] J. M. Jahangiri, Harmonic meromorphic starlike functions. Bulletin-Korean Mathematical Society, 37(2),2000, 291-302. 
Yusuf Abdulahi and Maslina Darus - On a Certain Class of Concave ...

[10] J. M. Jahangiri, Coefficient bounds and univalence criteria for harmonic functions with negative coefficients, Annales Universitatis Mariae Curie-Skłodowska 52(2), 1998, 57-66.

[11] I. Aldawish and M. Darus, . On Certain Class of Meromorphic Harmonic Concave Functions, Tamkang Journal of Mathematics 46(2), 2015 101-109.

[12] I. B. Jung, Y. C. Kim, and H. M. Srivastava, The Hardy space of analytic functions associated with certain one-parameter families of integral operators, J. Math. Anal. Appl. 176, (1993),138-147.

[13] K. O. Babalola,, Subclasses of Analytic Function Defined the Inverse integral operators, Analele Universitati Oradea. 1(2012),255-264.

Yusuf Abdulahi

Centre of Modelling and Data Science

Faculty of Science and Technology

Universiti Kebangsaan Malaysia.

Bangi 43600 Selangor.

email: yusuf.abdulai4success@gmail.com.

Maslina Darus

Centre of Modelling and Data Science

Faculty of Science and Technology

Universiti Kebangsaan Malaysia.

Bangi 43600 Selangor .

email: maslina@ukm.edu.my. 